\title{
PROTOCOLOS ASSISTENCIAIS COMO FERRAMENTA DE TRABALHO NO MANEJO CLÍNICO DA COVID-19 EM UNIDADE TERAPIA INTENSIVA: REVISÃO NARRATIVA
}

\section{ASSISTANCE PROTOCOLS AS A WORKING TOOL IN COVID-19 CLINICAL MANAGEMENT IN INTENSIVE THERAPY UNIT: NARRATIVE REVIEW}

\author{
Ellen Halitta Arantes ${ }^{1}$, Dinaelza Ribeiro da Rocha², Luciene dos Reis Pereira ${ }^{3}$, Júlio César Coelho do \\ Nascimento ${ }^{4}$
}

\author{
Submetido: $14 / 02 / 2021$ \\ Aprovado: 12/03/2021
}

\begin{abstract}
RESUMO
A infecção pelo Sars-CoV-2, causador da covid-19, se espalhou rapidamente devido ao alto nível de transmissibilidade, tornando-se uma pandemia mundial. Diante desse cenário, as medidas de prevenção são o principal aliado no combate ao vírus. Na UTI, local destina-se a receber pacientes em estado grave, é uma área conducente ao desenvolvimento de infecções relacionadas aos cuidados de saúde. Para prevenir e controlar essas infecções propõe-se a adesão aos protocolos assistenciais de prevenções recomendadas pelo Institute For Healthcare Improvement para auxiliar na organização do trabalho. Diante disso, o objetivo deste estudo consistiu em realizar uma revisão narrativa sobre os protocolos assistenciais como ferramenta de trabalho no manejo clínico da covid19 em UTI adulto. Esse tipo de revisão é fundamental na aquisição e atualização de conhecimento sobre uma temática específica. Os artigos foram extraídos das bases de dados: PubMed, BVS e EMBASE. A busca ocorreu em pares, em janeiro de 2021, de forma on-line, utilizando os descritores em Ciências da Saúde e o Medical Subject Headings: Intensive Care Units, Care Bundles, COVID19 virus e infection. A amostra final foi constituída de 6 artigos e estes foram discutidos com 0 suporte de outros estudos. Após análise dos dados, os resultados apontaram que a adesão aos pacotes de prevenção nas UTI auxilia na organização do trabalho e, diante desse cenário pandêmico deve ser intensificada para a segurança do paciente e do profissional a fim de promover um cuidado pautado na segurança e qualidade da assistência evitando diminuir as contaminações secundárias.
\end{abstract}

PALAVRA-CHAVE: Conjunto de intervenções. Covid-19. Terapia intensiva. Qualidade da Assistência à Saúde.

\begin{abstract}
Infection with Sars-CoV-2, which causes covid-19, spread rapidly due to the high level of transmissibility, causing a worldwide pandemic. Given this scenario, as measures to prevent the main problem of fighting the virus. In the ICU, the local destination is to receive patients in serious condition, it is an area conducive to the development of infections related to health care. To prevent and control these infections, it will apply to the preventive care protocols recommended by the Institute For Healthcare Improvement for auxiliary work organization. Therefore, the aim of this study was to carry out a narrative review of the care protocols as a work tool in the clinical laboratory of covid-19 in an adult ICU. This type of review is essential in acquiring and updating knowledge on a specific topic. The articles were extracted from the databases: PubMed, BVS and EMBASE. The search took place in pairs, in January 2021, online, using the descriptors in Health Sciences and the Medical Subject: Intensive Care Units, Care Packages, COVID-19 virus and infection. A final sample consisted of 6 articles and these were discussed with the support of other studies. After analyzing the

\footnotetext{
${ }^{1}$ Acadêmica de Enfermagem da Faculdade Padrão. E-mail: ellenhalitta@gmail.com

2 Acadêmica de Enfermagem da Faculdade Padrão. E-mail: dinaelzarocha17@gmail.com

${ }^{3}$ Acadêmica de Enfermagem da Faculdade Padrão. E-mail: lucienedosreispereira@gmail.com

4 Enfermeiro, Especialista em Oncologia Clínica, MBA em Serviço de Controle de Infecção Relacionado à Assistência em Saúde, Mestre em Assistência e Avaliação em Saúde, Docente do curso de Enfermagem da Faculdade Padrão e Faculdade Noroeste, Goiânia-GO. E-mail: prof.julioccnascimento@gmail.com
} 
PROTOCOLOS ASSISTENCIAIS COMO FERRAMENTA DE TRABALHO NO MANEJO CLÍNICO DA COVID-19 EM UNIDADE TERAPIA INTENSIVA: REVISÃO NARRATIVA Ellen Halitta Arantes, Dinaelza Ribeiro da Rocha, Luciene dos Reis Pereira, Júlio César Coelho do Nascimento

data, the results indicated for adherence to the prevention packages in the ICU auxiliary work organizations and, in view of this pandemic scenario, should be intensified for the safety of the patient and the professional in order to promote care based on safety and quality of care can decrease as secondary contamination.

KEYWORDS: Care Bundles. COVID-19 virus. Intensive Care Units. Quality of Health Care.

\section{INTRODUÇÃO}

O presente artigo é um recorte de um projeto de pesquisa maior, intitulado "Impact of prevention bundles on the clinical management of covid-19 in the Intensive Care Unit: systematic review and meta-analysis" cadastrado na plataforma International prospective register of systematic reviews (PROSPERO) sob registro no CRD42020181534, que visa a estudar o impacto dos bundles de prevenção no manejo clínico da covid-19 em Unidade de Terapia Intensiva (UTI), sobretudo no que se refere à aplicabilidade frente ao cenário pandêmico causado pelo vírus SARS-CoV-2.

Utilizou-se como referenciais teóricos autores que tratam sobre a temática, manuais da Society for Healthcare Epidemiology of America (SHEA), Institute For Healthcare Improvement (IHI), Ministério da Saúde (MS), Instituto Brasileiro para Segurança do Paciente (IBSP) e as recomendações da Associação de Medicina Intensiva Brasileira (AMIB).

Em dezembro de 2019, na cidade de Wuhan, província de Hubei, China, foi identificado o novo tipo de coronavírus, SARS-CoV-2 (Síndrome Respiratória Aguda Grave-Coronavírus-2), causador da doença covid-19 (Corona Virus Disease 2019) (GUAN et al. 2020). Logo após a identificação do vírus, a Organização Mundial da saúde (OMS), declarou a doença como emergência pública de interesse internacional.

A infecção pelo Sars-CoV-2 se espalhou rapidamente devido ao alto nível de transmissibilidade, tornando-se uma pandemia mundial. É um problema de emergência na saúde pública, principalmente devido à alta taxa de mortalidade e falta de opções de tratamento (PERLMAN, 2020).

Inicialmente a doença é caracterizada como uma síndrome gripal e apresenta como principais sintomas problemas respiratórios leves, febre persistente, em média de 5 a 6 dias após a infecção (LIMA, 2020). Em casos críticos, os pacientes podem apresentar dispneia grave, mialgia, confusão mental, cefaleia, odinofagia, rinorreia, dor torácica e em alguns casos podem apresentar episódios de diarreia, e náuseas e vômitos evidenciando a necessidade de cuidados intensivos na UTI (BRASIL, 2020).

Este local destina-se a receber pacientes em estado grave e, associado a inúmeros procedimentos invasivos, torna-se uma área crítica conducente ao desenvolvimento de infecções relacionadas aos cuidados de saúde. Diante desse cenário, as medidas de prevenção são o principal aliado no combate ao vírus. Para prevenir e controlar essas infecções propõe-se a adesão aos 


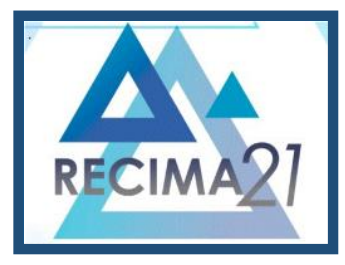

\section{RECIMA21 - REVISTA CIENTÍFICA MULTIDISCIPLINAR}

PROTOCOLOS ASSISTENCIAIS COMO FERRAMENTA DE TRABALHO NO MANEJO CLÍNICO DA COVID-19 EM UNIDADE TERAPIA INTENSIVA: REVISÃO NARRATIVA Ellen Halitta Arantes, Dinaelza Ribeiro da Rocha, Luciene dos Reis Pereira, Júlio César Coelho do Nascimento

bundles de prevenções recomendadas pelo Institute For Healthcare Improvement (IHI). Trata-se de protocolos assistenciais como medidas de prevenções direcionadas individualmente aos pacientes.

Considerando o alto nível de transmissibilidade do Sars-CoV-2, a adesão aos protocolos assistenciais como medidas de prevenção nas UTIs deve ser intensificada para a segurança do paciente e do profissional a fim de promover um cuidado pautado na segurança e qualidade da assistência.

Os protocolos assistenciais são tecnologias que fazem parte da organização do trabalho na área da saúde, sobretudo na assistência de enfermagem, e se constituem em uma importante ferramenta de gerenciamento (KRAUZER et al 2018). Neste cenário pandêmico, valer-se dessas tecnologias é extremamente importante para as instituições de saúde que prezam pela qualidade dos serviços assistenciais e buscam garantir a segurança dos profissionais e usuário. Na perspectiva de Foam et al (2016), a adoção dos protocolos para o cuidado em saúde é oportuno e auxilia na organização do trabalho. Diante disso, o objetivo deste estudo consistiu em realizar uma revisão narrativa sobre os protocolos assistenciais como ferramenta de trabalho no manejo clínico da covid19 em UTI adulto.

\section{MATERIAIS E MÉTODOS}

Para responder o objetivo proposto optou-se pelo estudo qualitativo de revisão narrativa, adequado para discutir o estado da arte de um determinado assunto. Trata-se de uma análise ampla da literatura, sem definir uma metodologia replicável conforme proposto por Vosgerau e Romanowsk (2014). Embora não apresente rigor metodológico, as revisões narrativas são fundamentais na aquisição e atualização de conhecimento sobre uma temática específica, evidenciando novas ideias conforme os registros apresentados na literatura (ROTHER, 2007).

A busca dos artigos ocorreu em pares, em janeiro de 2021, de forma on-line, utilizando os descritores em Ciências da Saúde (DeCS) e o Medical Subject Headings (MeSH): Intensive Care Units, Care Bundles, COVID-19 virus e infection. As bases de dados eletrônicas empregadas para seleção dos artigos foram: National Library of Medicine (PubMed), Biblioteca Virtual em Saúde (BVS) e The Excerpta Medica Database (EMBASE).

Em relação à seleção de estudos, foram avaliados de forma independente os trabalhos encontrados na busca eletrônica e, por meio de leitura dos títulos e resumos, os artigos duplicados foram excluídos. Em seguida foi realizada uma triagem para selecionar os estudos de acordo com os critérios de elegibilidade. Foram incluídos: artigos em qualquer idioma e qualquer ano de publicação em que o texto se relacionou aos protocolos assistenciais. Foram excluídos artigos que apresentaram estudos fora do contexto da UTI adulto. 


\section{RECIMA21 - REVISTA CIENTÍFICA MULTIDISCIPLINAR}

PROTOCOLOS ASSISTENCIAIS COMO FERRAMENTA DE TRABALHO NO MANEJO CLÍNICO DA COVID-19 EM UNIDADE TERAPIA INTENSIVA: REVISÃO NARRATIVA Ellen Halitta Arantes, Dinaelza Ribeiro da Rocha, Luciene dos Reis Pereira, Júlio César Coelho do Nascimento

Os estudos foram classificados quanto às particularidades da amostragem, agrupando aqueles cujas amostras foram de adultos sob cuidados em UTI. A partir daí, prosseguiu-se com a análise da fundamentação teórica dos estudos, bem como a observação das características gerais dos artigos.

Por fim, os dados apresentados foram submetidos a análise de conteúdo. Posteriormente, os resultados foram discutidos com o suporte de outros estudos provenientes dos manuais da Organização Mundial de Saúde (OMS), SHEA, IHI, Ministério da Saúde (MS), IBSP, Center for Disease Control and Prevention (CDC) e as recomendações da AMIB.

\section{RESULTADOS}

A Figura 1 apresenta os resultados das buscas nas bases de dados sobre o uso de protocolos assistências como ferramenta de trabalho no manejo clínico da covid-19 em UTI adulto.

Figura1. Fluxograma de seleção de artigos nas bases de dados

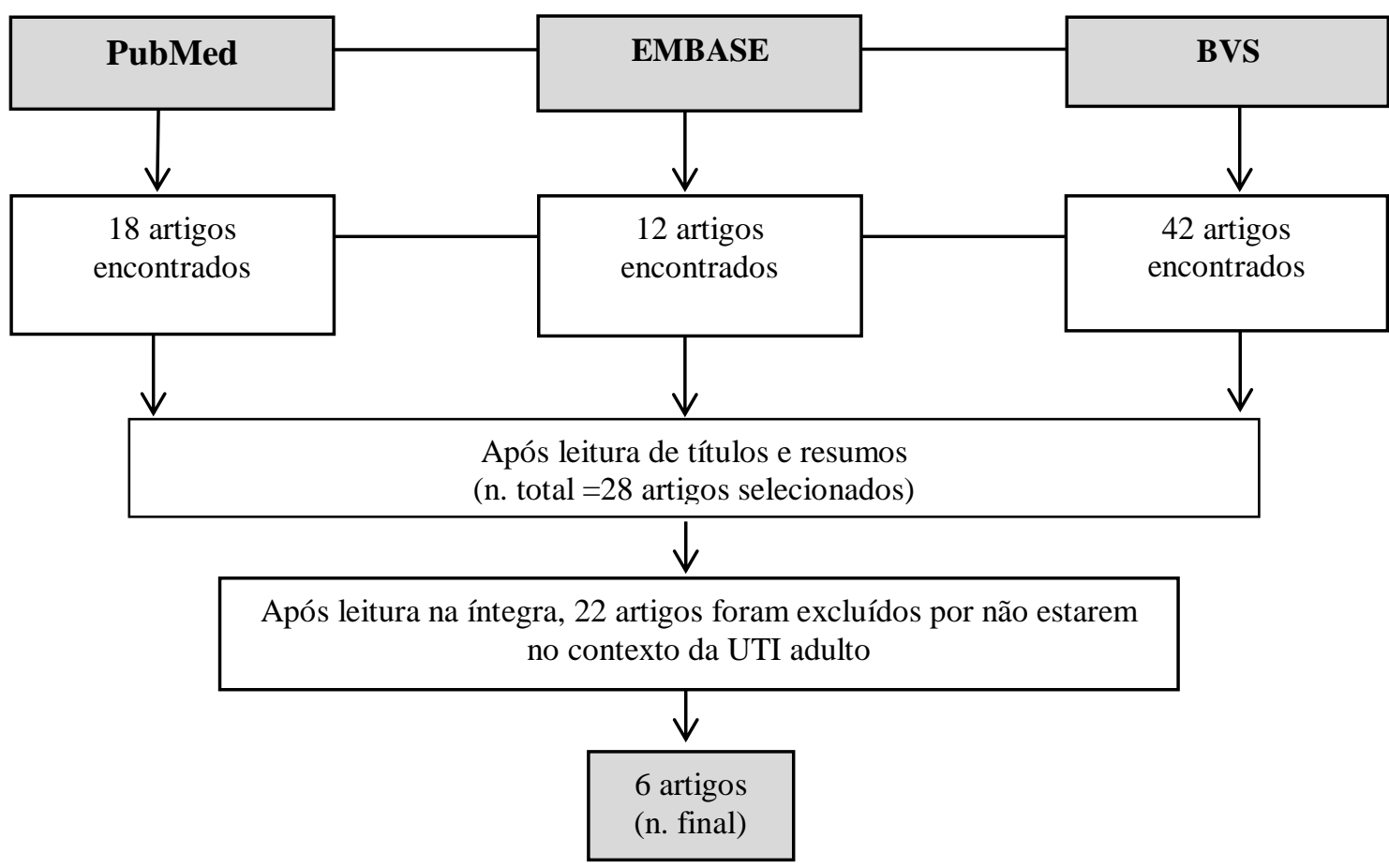

Fonte: autoria própria.

Dentre os estudos analisados, cincos relataram sobre a importância dos protocolos assistência para garantir a segurança do paciente e dos profissionais e destacaram também os 


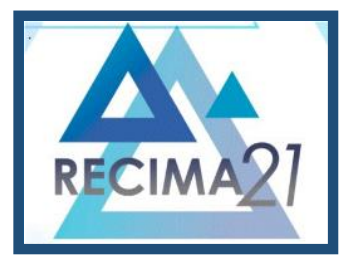

\section{RECIMA21 - REVISTA CIENTÍFICA MULTIDISCIPLINAR}

PROTOCOLOS ASSISTENCIAIS COMO FERRAMENTA DE TRABALHO NO MANEJO CLÍNICO DA COVID-19 EM UNIDADE TERAPIA INTENSIVA: REVISÃO NARRATIVA Ellen Halitta Arantes, Dinaelza Ribeiro da Rocha, Luciene dos Reis Pereira, Júlio César Coelho do Nascimento

diferentes tipos de EPI recomendados pelas agências nacionais e internacionais para 0 enfrentamento da Covid-19 no contexto da UTI (Tabela 1).

\begin{tabular}{llc}
\hline \multicolumn{1}{c}{ Tipos de EPI } & N & $\%$ \\
\hline Somente a máscara N95 & 1 & 16,6 \\
N95 ou PFF2 & 1 & 16,6 \\
N95 ou PFF3 + Face Shield & 2 & 33,3 \\
N95 + Face Shield ou óculos de proteção & 2 & 33,3 \\
Total & 6 & 100 \\
\hline
\end{tabular}

\section{DISCUSSÃO}

Os estudos encontrados foram unânimes em apontar o uso dos Equipamentos de proteção individual (EPI) como medida de segurança da equipe de saúde no atendimento de pacientes com covid-19 e a importância dos procedimentos operacionais padrão (POPs) no contexto da UTI adulto. Assim, de modo a organizar os resultados do levantamento dos artigos, optou-se por reuni-los em duas categorias que especificam os protocolos assistenciais como ferramenta de trabalho no manejo clínico da covid-19 em UTI adulto: 1) Equipamentos de proteção individual - segurança da equipe no atendimento de pacientes com Covid-19 e 2) Protocolos assistenciais utilizados na prática clínica no cenário da UTI.

\subsection{Equipamentos de proteção individual (EPI) - segurança da equipe no atendimento de pacientes com Covid-19}

A Norma Regulamentadora 6 (NR 6), da Portaria n. 3.214/78, do Ministério do Trabalho e Emprego, define EPI como "todo dispositivo ou produto, de uso individual utilizado pelo trabalhador, destinado a proteção de riscos suscetíveis que por virtude venha ameaçar a segurança e a saúde do colaborador" (FERREIRA; OLIVEIRA 2019)

Na prática clínica, segundo Simão et al (2010), os EPI's mais utilizados são: máscaras para proteção respiratória; óculos para proteger os olhos contra impactos, radiações e substancias; avental ou capote descartável e gorro para evitar aspersão de partículas dos cabelos e do couro cabeludo; luvas para proteger contra riscos biológicos e físicos. Além destes itens, atualmente, devido a pandemia, tem se incluído o uso dos protetores faciais, também chamados de Face Shield (figura 2). Trata-se de um equipamento que cobre a frente e as laterais do rosto e é recomendado 


\section{RECIMA21 - REVISTA CIENTÍFICA MULTIDISCIPLINAR}

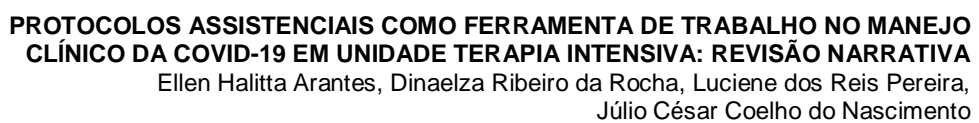

para todos os momentos de prestação de assistência, e principalmente se houver o risco de exposição dos profissionais a secreções corporais (WHO, 2020).

Figura 2. Face Shield.

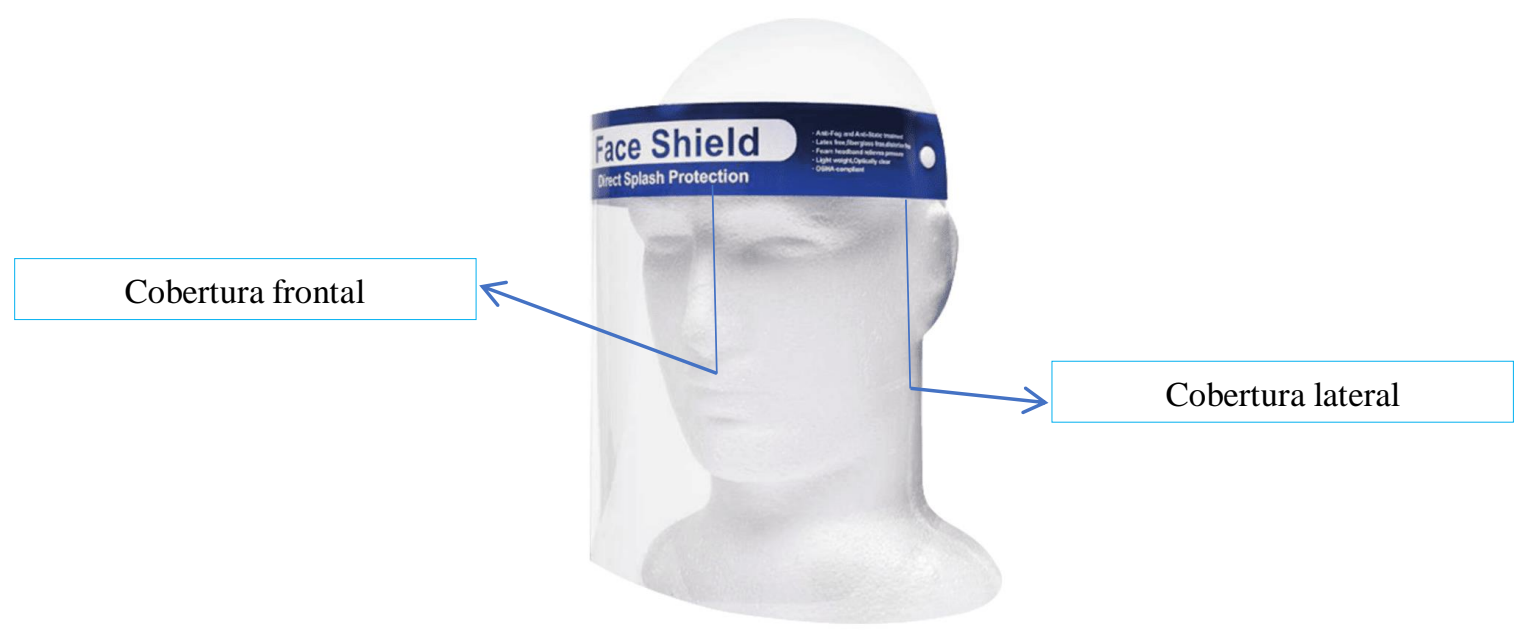

Fonte: https://www.prestigemedical.co.uk/product/medical-face-shield/ (adaptado pelos autores).

Sabe-se que o vírus SARS-CoV-2, causador da Covid-19 pode ser transmitido através de gotículas ou contato. Diante do grande potencial de transmissão e disseminação do vírus, os profissionais de saúde que prestam assistências diretas aos pacientes contaminados estão sujeitos ao adoecimento. Diante disso, é extremamente importante a adoção de medidas de prevenção de infecção a fim de diminuir as contaminações secundárias (SOUZA NETO; BORTOLUZZI; FREITAS, 2020).

No contexto da prevenção, o primeiro EPI a ser debatido são as máscaras de proteções respiratórias. Classificadas pela Agência Nacional de Vigilância Sanitária (ANVISA) (2020), em máscaras cirúrgicas e respiradores N-95 elas estão sendo amplamente utilizadas pela sociedade. No entanto, é importante ressaltar elas são recomendadas apenas para os profissionais de saúde em exercício da profissão, equipe de apoio que trabalhe a uma distância inferior a um metro dos pacientes diagnosticados ou suspeitos por infecção pelo SARS-CoV-2, bem como para o próprio paciente e seu acompanhante (SOUZA NETO; BORTOLUZZI; FREITAS, 2020).

As máscaras cirúrgicas, segundo Franco et al (2020), devem ser confeccionadas de material tecido-não tecido (TNT), possuir no mínimo uma camada interna e outra externa e obrigatoriamente, um elemento filtrante.

"A camada externa e o elemento filtrante, para serem eficientes, devem ser resistentes à penetração de fluidos transportados pelo ar. Além disso, tudo, ela 


\section{RECIMA21 - REVISTA CIENTÍFICA MULTIDISCIPLINAR}

PROTOCOLOS ASSISTENCIAIS COMO FERRAMENTA DE TRABALHO NO MANEJO CLÍNICO DA COVID-19 EM UNIDADE TERAPIA INTENSIVA: REVISÃO NARRATIVA Ellen Halitta Arantes, Dinaelza Ribeiro da Rocha, Luciene dos Reis Pereira, Júlio César Coelho do Nascimento

precisa cobrir a área do nariz e boca e ter um ajuste adequado do contorno do nariz e das bochechas"

Devido ao uso de forma geral, atualmente os EPI estão cada vez mais escassos. Diante desse cenário, o Ministério da Saúde recomenda o reaproveitamento das máscaras N95, N99, N100, PFF2 ou PFF3 em situações de integridade, limpas e com vedação adequada. É importante ressaltar que a reutilização só é recomendada para o mesmo usuário, portanto, não deve ser compartilhada (BRASIL, 2020). Estas devem ser utilizadas apenas em procedimentos que geram aerossóis, tais como: intubação ou aspiração traqueal; ventilação mecânica invasiva e não invasiva; ressuscitação cardiopulmonar; ventilação manual antes da intubação e coletas de amostras nasotraqueais.

\subsection{Protocolos assistenciais utilizados na prática clínica no cenário da UTI}

Os Protocolos assistenciais, comumente conhecidos como Procedimentos Operacionais Padrão (POP), é um documento organizacional que auxilia no planejamento do trabalho a ser executado. Ele deve conter instruções detalhadas para alcançar a uniformidade na aplicação de uma determinada função (KRAUZER et al, 2018).

Nesse sentido, a adoção dos protocolos assistenciais para o cuidado é apropriado e dá sustentação para organização e gerenciamento do trabalho de enfermagem e demais áreas da saúde (CRUZ et al, 2016)

Considerando que o cenário da pandemia se modifica constantemente é extremamente importante revisar de forma contínua os protocolos assistenciais utilizados para combater a COVID19, principalmente no contexto da UTI (SILVA et al, 2020).

Atualmente os protocolos estão voltados para a paramentação e desparamentação dos EPIs utilizados pelos profissionais. Acredita-se que a contaminação está ligada diretamente no momento da desparamentação. Por esse motivo, a ANVISA (2020) elaborou um informativo para orientar os profissionais de saúde neste momento (quadro 1).

Quadro 1. Recomendações para desparamentação.

\begin{tabular}{|l|}
\hline Ainda dentro do quarto/ box do paciente com Covid-19 \\
\hline 1 Retirar as luvas \\
\hline 2 Retirar o avental \\
\hline 3 Higienizar as mãos \\
\hline Sair do quarto/ box onde se encontra o paciente com Covid-19 \\
\hline 1 Higienizar as mãos \\
\hline 2 Retirar gorro \\
\hline 3 Retirar óculos de proteção ou Face Shield \\
\hline
\end{tabular}




\section{RECIMA21 - REVISTA CIENTÍFICA MULTIDISCIPLINAR}

PROTOCOLOS ASSISTENCIAIS COMO FERRAMENTA DE TRABALHO NO MANEJO CLÍNICO DA COVID-19 EM UNIDADE TERAPIA INTENSIVA: REVISÃO NARRATIVA Ellen Halitta Arantes, Dinaelza Ribeiro da Rocha, Luciene dos Reis Pereira, Júlio César Coelho do Nascimento

\begin{tabular}{|l|}
\hline 4 Higienizar as mãos \\
\hline 5 Retirar máscara N95/PFF2 \\
\hline 6 Higienizar as mãos. \\
\hline
\end{tabular}

Fonte:https://www20.anvisa.gov.br/segurancadopaciente/index.php/publicacoes/item/desparamentac ao-de-epis?category id=245 (adaptado pelos autores)

\section{CONSIDERAÇÕES FINAIS}

Os protocolos assistenciais como ferramenta de trabalho no manejo clínico da Covid-19 em UTI adulto, são extremamente importantes para evitar a contaminação do profissional durante a assistência prestada ao paciente.

Evidenciou-se que os protocolos voltados para o uso de EPIs são essenciais no âmbito hospitalar, cada tipo de EPI possui a sua indicação de uso, mas é fato que diante da atual problemática ocasionada pela pandemia de COVID19 faz-se necessário que os profissionais de saúde se mantenham protegidos, atualizados e trabalhem em conformidade com as recomendações nacionais e internacionais de saúde, não se esquecendo de seguir também as outras formas de prevenção já existente antes da pandemia.

\section{REFERÊNCIAS}

AGÊNCIA NACIONAL DE VIGILÂNCIA SANITÁRIA. ANVISA. Orientações gerais: racionalização do uso de respiradores descartáveis nos estabelecimentos fabricantes de medicamentos, insumos farmacêuticos, produtos para saúde, cosméticos e saneantes. Brasília, DF: ANVISA, 2020. Disponível em: https://www.gov.br/anvisa/pt-br. Acesso em: 13 jan. 2021.

AGÊNCIA NACIONAL DE VIGILÂNCIA SANITÁRIA. ANVISA. Desparamentação Equipamentos de Proteção Individual (EPI) utilizados em procedimentos geradores de aerossóis. Disponível em: https://www20.anvisa.gov.br/segurancadopaciente/index.php/publicacoes/item/desparamentacao-deepis?category id=245. Acesso em: 13 jan. 2021.

CENTERS FOR DISEASE CONTROL AND PREVENTION. Using Personal Protective Equipment (PPE). Atlanta: CDC, 2020. Disponível em: https://www.cdc.gov/coronavirus/2019- ncov/hcp/usingppe.html. Acesso em: 09 jan. 2021.

CRUZ, F. O. A. M.; FERREIRA, E. B.; VASQUES, C. I. et al. Validation of an educative manual for patients with head and neck cancer submitted to radiation therapy. Rev Latino-Am Enferm, v. 24, p. e2706, 2016.

FERREIRA, W. F. S.; OLIVEIRA, E, M. Biossegurança em relação a adesão de equipamentos de proteção individual. Revista da Universidade Vale do Rio Verde, Betim - MG, v. 17, n.1, p. 1-9, 2019.

GUAN, W.; NI, Z.; YU HU, W. et al. Clinical Characteristics of Coronavirus Disease 2019 in China. $\mathbf{N}$ Engl J Med, Boston - EUA, v. 382, p.1708-1720, 2020. DOI: 10.1056/NEJMoa2002032. 


\section{RECIMA21 - REVISTA CIENTÍFICA MULTIDISCIPLINAR}

PROTOCOLOS ASSISTENCIAIS COMO FERRAMENTA DE TRABALHO NO MANEJO
CLÍNICO DA COVID-19 EM UNIDADE TERAPIA INTENSIVA: REVISÃO NARRATIVA
Ellen Halitta Arantes, Dinaelza Ribeiro da Rocha, Luciene dos Reis Pereira,
Júlio César Coelho do Nascimento

KRAUZER, I. M.; DALL'AGNOLL, C. M.; GELBCKE, F. L. et al. A construção de protocolos assistenciais no trabalho em Enfermagem. REME - Rev Min Enferm, v. 22, p. e-1087, 2018. DOI: $10.5935 / 1415-2762.20180017$

LIMA, C. M. A. O. Informações sobre o novo coronavírus (COVID-19). Radiol Bras. São Paulo, v. 53, n. 2, p. V-VI, 2020. DOI: https://doi.org/10.1590/0100-3984.2020.53.2e1.

NASCIMENTO, M. N. R.; SANTOS, A. G.; BARROS, L. A. F.; OLIVEIRA, C. J. Cuidados de enfermagem na proteção e prevenção de riscos para o enfermeiro: revisão da literatura. J. nurs. Health, v. 10, n. Esp, p. e20104001, 2020.

PERLMAN, S. Another Decade, Another Coronavirus. N Engl J Med, Boston - EUA, v. 382, p. 760762, 2020. DOI: 10.1056/NEJMe2001126.

ROTHER, E. T. Revisão sistemática X revisão narrativa. Acta paul. Enferm, v. 20, n. 2, p. V-VI, 2007.

SILVA, D. M.; RODRIGUES, N. H.; SILVA, L. G. A. et al. Recomendações para a utilização de máscara em ambiente hospitalar durante a pandemia ocasionada pelo Coronavírus. J. nurs. health. v. 10, n. Esp., p. e20104010, 2020.

SIMÃO, S. A. F. et al. Acidentes de trabalho com material perfurocortante envolvendo profissionais de enfermagem de unidade de emergência hospitalar. Rev. enferm. UERJ, v. 18, n. 3, p. 400-4, 2010.

VOSGERAU, D. S. A. R.; ROMANOWSKI, J. P. Estudos de revisão: implicações conceituais e metodológicas. Revista de Diálogo Educacional, v. 14, n. 41, p. 165-189, 2014.

WORLD HEALTH ORGANIZATION. Rational use of personal protective equipment (PPE) for coronavirus disease (COVID-19): interim guidance, 19 March 2020. Geneva: WHO; 2020. Disponível em: https://apps.who.int/iris/handle/10665/331498. Acesso em: 13 jan. 2021. 